TRANSACTIONS OF THE

AMERICAN MATHEMATICAL SOCIETY

Volume 327, Number 2, October 1991

\title{
CONVEX OPTIMIZATION AND THE EPI-DISTANCE TOPOLOGY
}

\author{
GERALD BEER AND ROBERTO LUCCHETTI
}

\begin{abstract}
Let $\Gamma(X)$ denote the proper, lower semicontinuous, convex functions on a Banach space $X$, equipped with the completely metrizable topology $\tau$ of uniform convergence of distance functions on bounded sets. A function $f$ in $\Gamma(X)$ is called well-posed provided it has a unique minimizer, and each minimizing sequence converges to this minimizer. We show that well-posedness of $f \in \Gamma(X)$ is the minimal condition that guarantees strong convergence of approximate minima of $\tau$-approximating functions to the minimum of $f$. Moreover, we show that most functions in $\left\langle\Gamma(X), \tau_{a w}\right\rangle$ are well-posed, and that this fails if $\Gamma(X)$ is topologized by the weaker topology of Mosco convergence, whenever $X$ is infinite dimensional. Applications to metric projections are also given, including a fundamental characterization of approximative compactness.
\end{abstract}

\section{INTRODUCTION}

Let $\mathscr{C}(X)$ (resp. $\left.\mathscr{C}_{B}(X)\right)$ be the closed (resp. closed and bounded) nonempty convex sets in a normed linear space $X$. For over a half-century the basic topology on $\mathscr{C}_{B}(X)$ has been the well-known Hausdorff metric topology [15]. How should this topology be extended to $\mathscr{C}(X)$ ? The generally recognized $[2,37]$ successful solution in finite dimensions is the completely metrizable Fell topology, generated by all sets of the form $V^{-} \equiv\{A \in \mathscr{C}(X): A \cap V \neq \varnothing\}$ where $V$ is open in $X$, and $\left(K^{c}\right)^{+} \equiv\left\{A \in \mathscr{C}(X): A \subset K^{c}\right\}$ where $K$ is a compact subset of $X$. Convergence of a sequence $\left\langle A_{n}\right\rangle$ to $A$ in this topology in finite dimensions is equivalent to classical Kuratowski convergence of sets [27, §29]; alternatively, it is equivalent to the pointwise convergence of the associated sequence of distance functions $\left\langle d\left(\cdot, A_{n}\right)\right\rangle$ to $d(\cdot, A)$ (see, e.g., [11, 20]).

Certainly one of the most important features of the Fell topology on $\mathscr{C}(X)$ is its stability with respect to duality, as established by Wijsman [41], expressed by the continuity of the polar map $A \rightarrow A^{\circ}$, or in the case of proper lower

Received by the editors April 20, 1989 and, in revised form, September 5, 1989.

1980 Mathematics Subject Classification (1985 Revision). Primary 49B50, 26B25; Secondary 54B20, 41A50.

Key words and phrases. Convex optimization, convex function, well-posed minimization problem, epi-distance topology, Mosco convergence, metric projection, approximative compactness, Chebyshev set.

Research of the second author was partially supported by C.N.R. 
semicontinuous convex functions as identified with their epigraphs, by the continuity of the conjugate map $f \rightarrow f^{*}$. Convergence of functions in this sense is called epiconvergence in the literature.

Intense efforts over the past twenty years have been focused on extending the basic results about the Fell topology for convex sets to infinite dimensions, requiring the introduction of topologies/convergence notions on $\mathscr{C}(X)$ which, in finite dimensions, reduce to convergence with respect to the Fell topology. Most prominent in this endeavor were the pioneering papers of Mosco [32, 33], where the definition of Kuratowski convergence for sequences of convex sets was modified in a most natural way: a sequence $\left\langle A_{n}\right\rangle$ in $\mathscr{C}(X)$ is declared Mosco convergent to $A \in \mathscr{C}(X)$ provided both of the following conditions are met:

(i) for each $a \in A$, there exists a sequence $\left\langle a_{n}\right\rangle$ strongly convergent to $a$ such that for each $n$, we have $a_{n} \in A_{n}$;

(ii) whenever $n(1)<n(2)<\cdots$, and whenever $a_{k} \in A_{n(k)}$ for each $k \in Z^{+}$, then the weak convergence of $\left\langle a_{k}\right\rangle$ to $x \in X$ implies $x \in A$.

Mosco successfully extended Wijsman's sequential continuity results to the reflexive setting [33] (see also [8, 25]), but Mosco convergence is not stable with respect to duality in an arbitrary Banach space [10]. Moreover, Mosco convergence does not reduce to Hausdorff metric convergence on $\mathscr{C}_{B}(X)$ (in $l_{2}$, let $A_{n}$ be the line segment joining the origin to $e_{n}$ ). The first tractable topology on $\mathscr{C}(X)$ stable with respect to duality $[10,16]$ in a general normed linear space that reduces to the Fell topology in finite dimensions has been seriously studied only recently $[3,4,5,6,9,10,16]$. Convergence of a sequence $\left\langle A_{n}\right\rangle$ to $A$ in this topology means nothing more than uniform convergence of $\left\langle d\left(\cdot, A_{n}\right)\right\rangle$ to $d(\cdot, A)$ on bounded subsets of $X$. Uniform convergence of distance functions on bounded sets is compatible with a completely metrizable topology, provided $X$ is complete (see $\S 2$ below). Although this convergence notion can be found in disguise in [32], we denote it by $\tau_{a w}$ in the sequel, in recognition of its development by Attouch and Wets for spaces of functions. Following these authors, we call $\tau_{a w}$ when restricted to the proper, lower semicontinuous, convex functions $\Gamma(X)$ on a normed linear space $X$ the epi-distance topology.

In this paper we study convex minimization problems and the topology $\tau_{a w}$. In many such problems, one is forced to approximate a given objective function $f \in \Gamma(X)$ by more tractable perturbed functions $\left\langle f_{n}\right\rangle$. Ideally, one would hope that minima (or approximate minima) of the perturbed sequence would converge to a minimum of $f$, and that $\inf _{X} f=\lim _{n \rightarrow \infty} \inf _{X} f_{n}$, as $\left\langle f_{n}\right\rangle$ converges to $f$. We show that when convergence means $\tau_{a w}$-convergence, this behavior is guaranteed if and only if $f$ is well-posed [40, 21, 29]: $f$ has a unique minimizer $x_{0}$, and each minimizing sequence for $f$ converges to $x_{0}$. Furthermore, we show that $\left\langle\Gamma(X), \tau_{a w}\right\rangle$ is completely metrizable whenever $X$ is a Banach space, and that most convex functions (in the sense of Baire category) are well-posed. All of the above results fail for the topology of Mosco 
convergence. Finally, we obtain some basic results about metric projections, showing that in any reflexive space, most elements of $\left\langle\mathscr{C}(X), \tau_{a w}\right\rangle$ are Chebyshev, and that for most $(x, A) \in X \times \mathscr{C}(X)$, the associated metric projection program is well-posed.

\section{Preliminaries}

In the sequel we denote the unit ball and origin of our normed linear space $X$ by $U$ and $\theta$, respectively. $X^{*}$ will represent the continuous dual of $X$, with dual unit ball $U^{*}$ and origin $\theta^{*}$. We equip $X \times R$ with the box norm: $\|(x, \alpha)\|=\max \{\|x\|,|\alpha|\}$. If $f: X \rightarrow[-\infty, \infty]$ is any function, its epigraph is the following subset of $X \times R$ : epi $f=\{(x, \alpha): x \in X, \alpha \in R$, and $\alpha \geq f(x)\}$. If epi $f \neq \varnothing$ and contains no vertical lines, we call $f$ proper; $f$ is convex (respectively lower semicontinuous) provided epi $f$ is a convex (resp. closed) subset of $X \times R$. We write $v(f)$ for $\inf \{f(x): x \in X\}$, and $\arg \min f$ for the possibly empty set of points $\{x \in X: f(x)=v(f)\}$. For each $\alpha \in R$, we denote by $\operatorname{lev}(f ; \alpha)$ the sublevel set of $f$ at height $\alpha$, that is, $\{x \in X: f(x) \leq \alpha\}$. Convexity (resp. lower semicontinuity) of $f$ guarantees that each sublevel set is convex (resp. closed).

Again, $\Gamma(X)$ will denote the proper, lower semicontinuous, convex functions on $X$. If $A \in \mathscr{C}(X)$ we denote the distance function for $A$ by $d(\cdot, A)$. It is standard to identify $A \in \mathscr{C}(X)$ with its indicator function $I(\cdot, A) \in \Gamma(X)$, defined by

$$
I(x, A)= \begin{cases}0, & \text { if } x \in A, \\ \infty, & \text { if } x \notin A .\end{cases}
$$

For each $f \in \Gamma(X)$ its conjugate $f^{*} \in \Gamma\left(X^{*}\right)$ is defined by the familiar formula $f^{*}(y)=\sup \{\langle x, y\rangle-f(x): x \in X\}$. All of the above terminology is standard (see, e.g., $[15,23,24,36])$.

We now turn to a discussion of $\tau_{a w}$ and the topology of Mosco convergence $\tau_{M}$ on $\mathscr{C}(X)$. These in turn give rise to topologies on $\Gamma(X)$, with functions identified with their epigraphs. On $\mathscr{E}(X)$, uniform convergence of distance functions on bounded subsets of $X$ is formally convergence with respect to a topology on $\mathscr{C}(X)$, induced by a uniformity on $\mathscr{C}(X)$ with a (countable) base consisting of the following sets:

$$
\Omega[n]=\left\{(A, B): \sup _{\|x\| \leq n}|d(x, A)-d(x, B)|<1 / n\right\} \quad\left(n \in Z^{+}\right) .
$$

Denoting $\sup _{\|x\| \leq n}|d(x, A)-d(x, B)|$ by $d_{n}(A, B)$, it is clear that the induced topology is defined by the following metric on $\mathscr{C}(X)$ :

$$
m(A, B)=\sum_{n=1}^{\infty} 2^{-n}\left[d_{n}(A, B) /\left(1+d_{n}(A, B)\right)\right] .
$$

By Theorem 2.1 of [3], this metric is complete if $X$ is a Banach space. We remark that in finite dimensions, uniform convergence of distance functions 
on bounded subsets is no stronger than their pointwise convergence, because distance functions are equicontinuous.

Recall [15] that the excess of a set $A$ over a set $B$ is defined by the formula $e(A, B)=\inf \{\varepsilon>0: A \subset B+\varepsilon U\}$, and that the Hausdorff distance between $A$ and $B$ is given by haus $(A, B)=\max \{e(A, B), e(B, A)\}$. Since the Hausdorff distance between two sets $A$ and $B$ is nothing but $\sup _{x \in X}|d(x, A)-d(x, B)|$, it is not surprising that $\tau_{a w}$ admits a presentation akin to the standard presentation of Hausdorff distance. For each $\rho>0$, we define the $\rho$-Hausdorff distance $[3,4,10]$ between $A$ and $B$ by the formula

$$
\operatorname{haus}_{\rho}(A, B)=\max \{e(A \cap \rho U, B), e(B \cap \rho U, A)\} \text {. }
$$

Apparently, the connection between $\tau_{a w}$ and the "distances" \{haus $\left.\rho: \rho>0\right\}$ was first observed in [6] and in [9]: $A=\tau_{a w}-\lim A_{n}$ if and only if for each $\rho>$ 0 , we have $\lim _{n \rightarrow \infty}$ haus $\rho\left(A_{n}, A\right)=0$. Actually, to show that $A=\tau_{a w}-\lim A_{n}$, we need only show that $\lim _{n \rightarrow \infty}$ haus ${ }_{\rho}\left(A_{n}, A\right)=0$ for all $\rho$ beyond some fixed $\rho_{0}$ because haus $\rho$ increases with $\rho$. We will use this fact repeatedly. From the perspective of uniformities, the connection between $\tau_{a w}$ and \{haus $\rho: \rho>$ 0) may be recast as follows [3, Theorem 1.2]: a (countable) base for another (weaker!) compatible uniformity for $\tau_{a w}$ consists all sets of the form:

$$
\Sigma[n]=\left\{(A, B): \operatorname{haus}_{n}(A, B)<1 / n\right\} \quad\left(n \in Z^{+}\right) .
$$

Given that Mosco convergence was introduced twenty years ago and has been of great interest thereafter [2,39], it is inexplicable why a simple topology compatible with Mosco convergence in any Banach space [6, Theorem 3.1] was not identified until very recently. This Mosco topology $\tau_{M}$ has as a subbase all sets of the form:

$$
\begin{gathered}
V^{-} \equiv\{A \in \mathscr{C}(X): A \cap V \neq \varnothing\} \quad(V \text { norm open }) \\
\left(K^{c}\right)^{+} \equiv\left\{A \in \mathscr{C}(X): A \subset K^{c}\right\} \quad(K \text { weakly compact }) .
\end{gathered}
$$

Like Mosco convergence, this topology is well-behaved only when $X$ is reflexive. In this setting, it is Hausdorff and completely regular, but it is metrizable if and only if $X$ is separable [7]. Evidently, this topology reduces to the Fell topology in finite dimensions. As noted earlier, the Fell topology and the topology of pointwise convergence of distance functions agree in finite dimensions; so, $\tau_{M}=\tau_{a w}$ here. The Mosco topology $\tau_{M}$ is in general weaker than $\tau_{a w}[4$, Proposition 4.5 and 10, Lemma 2.1], and in reflexive spaces, stronger than the topology of pointwise convergence of distance functions [7, Theorem 3.5; 39, p. II. 6]. That $\tau_{M}$-convergence need not guarantee $\tau_{a w}$-convergence is easy to see: in $l_{2}$, again let $A_{n}=\operatorname{conv}\left\{\theta, e_{n}\right\}$. That pointwise convergence of distance functions in reflexive spaces need not guarantee $\tau_{M}$-convergence is not easy to see, and requires that the dual norm for $X^{*}$ fails to have the Kadec property $[9,14]$. 
It is very well known $[2,32]$ that $\tau_{M}$-convergence of a sequence $\left\langle f_{n}\right\rangle$ in $\Gamma(X)$ to $f \in \Gamma(X)$ is equivalent to the conjunction of the following two conditions:

(a) for each $x \in X$, there exists a sequence $\left\langle x_{n}\right\rangle$ strongly convergent to $x$ for which $\lim _{n \rightarrow \infty} f_{n}\left(x_{n}\right)=f(x)$;

(b) for each $x \in X$, whenever $\left\langle x_{n}\right\rangle$ is weakly convergent to $x$, we have $f(x) \leq \liminf _{n \rightarrow \infty} f_{n}\left(x_{n}\right)$.

For reflexive spaces, the conjugate map $f \rightarrow f^{*}$ is a homeomorphism of $\left\langle\Gamma(X), \tau_{M}\right\rangle$ onto $\left\langle\Gamma\left(X^{*}\right), \tau_{M}\right\rangle$ [8]; this is also true if the function spaces are equipped with the stronger epi-distance topology [4], even without reflexivity $[10,16]$. More fundamentally, $\tau_{M}$ is the weakest topology on $\Gamma(X)$ such that the epigraphical multifunctions $f \rightarrow$ epi $f$ and $f \rightarrow$ epi $f^{*}$ are both lower semicontinuous $[27, \S 18]$ as set-valued functions [12].

\section{SOME TOOL THEOREMS FOR $\tau_{a w}$-CONVERGENCE}

In this section we collect some basic facts about $\tau_{a w}$-convergence of sets and functions that have not appeared in the literature. We will apply all of them in subsequent sections. In the process, we point out the favorable properties of this convergence that distinguish it from the less well-behaved Mosco convergence.

Evidently, a uniformly bounded sequence in $\mathscr{C}(X)$ is $\tau_{a w}$-convergent if and only if it is convergent in Hausdorff distance. In fact, this equivalence holds assuming only that the limit set is bounded, a fact noted by Salinetti and Wets in finite dimensions [38]. As a result, the topology $\tau_{a w}$ reduces to the usually stronger Hausdorff metric topology when restricted to the bounded elements of $\mathscr{C}(X)$.

Lemma 3.1. Let $X$ be a normed linear space, and let $A \in \mathscr{C}(X)$ be bounded. Suppose $\left\langle A_{n}\right\rangle$ is a sequence in $\mathscr{C}(X) \tau_{\text {aw }}$-convergent to $A$. Then $\left\langle A_{n}\right\rangle$ is convergent to $A$ in Hausdorff distance.

Proof. Fix $a_{0} \in A$ and choose $\rho>0$ with $A \subset \rho U$. By the definition of $\tau_{a w}$, there exists $N \in Z^{+}$such that for each $n>N$ we have

$$
\sup \left\{\left|d(x, A)-d\left(x, A_{n}\right)\right|:\|x\| \leq \rho+3\right\}<1 .
$$

We claim that for all $n>N$, we have $A_{n} \subset(\rho+2) U$. Suppose not; then there exists $a_{n} \in A_{n}$ with $\left\|a_{n}\right\|>\rho+2$. Since $d\left(a_{0}, A_{n}\right)<1$, there exists $b_{n} \in A_{n}$ with $\left\|b_{n}\right\|<\rho+1$. As a result, some convex combination $c_{n}$ of $a_{n}$ and $b_{n}$ has norm $\rho+2$ so that $\left\|d\left(c_{n}, A_{n}\right)-d\left(c_{n}, A\right)\right\|=d\left(c_{n}, A\right) \geq 2$, a contradiction to the choice of $N$. Thus, $\left\langle A_{n}\right\rangle$ is uniformly bounded eventually, and converges to $A$ in Hausdorff distance.

We remark in passing that Lemma 3.1 remains valid for sequences of connected sets but fails in general. As an immediate corollary we have

Lemma 3.2. Let $X$ be a normed linear space; then $A \rightarrow \operatorname{diam} A$ is a continuous extended real valued functional on $\left\langle\mathscr{E}(X), \tau_{a w}\right\rangle$. 
Proof. Fix $A_{0} \in \mathscr{C}(X)$ and let $\left\langle A_{n}\right\rangle$ be a sequence in $\mathscr{C}(X) \tau_{a w}$-convergent to $A_{0}$. We consider two cases: (i) $\operatorname{diam} A_{0}=\infty$; (ii) $\operatorname{diam} A_{0}<\infty$.

In case (i), upper semicontinuity of the diameter functional obviously holds at $A_{0}$. For lower semicontinuity, we show that for each $\alpha>0$, there exists $N \in Z^{+}$such that for each $n>N$ we have $\operatorname{diam} A_{n}>\alpha$. Choose points $a$ and $b$ in $A_{0}$ with $\|a-b\|>\alpha$. Let $\varepsilon=(\|a-b\|-\alpha) / 2$ and let $\rho=\max \{\|a\|,\|b\|\}$. There exists $N \in Z^{+}$with haus ${ }_{\rho}\left(A_{n}, A\right)<\varepsilon$ for each $n>N$. For each such $n$, there exist $a_{n}$ and $b_{n}$ in $A_{n}$ with $\left\|a_{n}-a\right\|<\varepsilon$ and $\left\|b_{n}-b\right\|<\varepsilon$, and as a result, $\operatorname{diam} A_{n}>\alpha$. In case (ii), by Lemma 3.1, we have convergence of $\left\langle A_{n}\right\rangle$ to $A_{0}$ in Hausdorff distance, where continuity of the diameter functional is well known.

Consideration of the example in $l_{2}$ presented in the introduction shows that Lemma 3.2 fails with $\tau_{M}$-convergence replacing $\tau_{a w}$-convergence. More precisely, on $\left\langle\mathscr{C}(X), \tau_{M}\right\rangle, A \rightarrow \operatorname{diam} A$ is only lower semicontinuous.

We use the next fact twice in the sequel.

Lemma 3.3. Let $\left\langle A_{n}\right\rangle$ be an increasing sequence of closed (convex) subsets of $a$ normed linear space $X$ such that for each $\rho>0$ there exists $n$ with $\rho U \subset A_{n}$. Then for each $A \in \mathscr{C}(X)$, we have $A=\tau_{a w}-\lim A_{n} \cap A$.

Proof. For each $\rho>0$, there exists $N \in Z^{+}$such that for all $n>N$, we have $A \cap \rho U=A_{n} \cap A \cap \rho U$. Thus, haus ${ }_{\rho}\left(A, A_{n} \cap A\right)=0$ for each $n>N$.

The next lemma may be found buried in the proof of Theorem 11 of [31]. Although it is very simple, it is, in our view, an important technical feature of $\tau_{a w}$-convergence. It is an immediate consequence of the following version of the Radström cancellation principle [6], which itself follows easily from the separation theorem.

Radström cancellation principle. Let $A, B$, and $C$ be closed convex subsets of a normed linear space $X$ with $B$ bounded. Suppose $A+B \subset C+B$. Then $A \subset C$.

Lemma 3.4. Let $X$ be a normed linear space. Suppose $B \in \mathscr{C}(X), C \in \mathscr{C}(X)$, and haus ${ }_{\rho}(C, B)<\delta$. Then whenever $x+2 \delta U \subset B \cap \rho U$, we have $x+\delta U \subset C$.

Proof. We have the following inclusions:

$$
(x+\delta U)+\delta U=x+2 \delta U \subset B \cap \rho U \subset C+\delta U .
$$

By the Radström cancellation principle, this yields $x+\delta U \subset C$.

An immediate consequence of Lemma 3.4 is that $\{A \in \mathscr{C}(X)$ : int $A \neq \varnothing\}$ is $\tau_{a w}$-open. This fails for the Mosco topology.

Example. In $l_{2}$, the unit ball is the $\tau_{M}$-limit of $\left\langle A_{n}\right\rangle$ where for each $n$

$$
A_{n}=\left\{x: \sum_{i=1}^{n}\left\langle x, e_{i}\right\rangle^{2} \leq 1 \text { and }\left\langle x, e_{i}\right\rangle=0 \text { for } i>n\right\} \text {. }
$$

Evidently, each $A_{n}$ has empty interior. 
We now turn our attention to $\Gamma(X)$.

Lemma 3.5. Let $X$ be a Banach space. Then $\left\langle\Gamma(X), \tau_{a w}\right\rangle$ is completely metrizable.

Proof. As mentioned in $\S 2$, the space $\left\langle\mathscr{C}(X \times R), \tau_{a w}\right\rangle$ is completely metrizable. Evidently, the lower semicontinuous convex functions on $X$ (other than the function that is identically equal to $\infty$ ) may be described as the following set: $\mathscr{F} \equiv\{A \in \mathscr{C}(X \times R)$ : whenever $(x, \alpha) \in A$ and $\beta>\alpha$, then $(x, \beta) \in A\}$. It is routine to check that $\mathscr{F}$ is $\tau_{a w}$-closed in $\mathscr{C}(X \times R)$, so that $\left\langle\mathscr{F}, \tau_{a w}\right\rangle$ is completely metrizable. Since a closed convex set in $X \times R$ that contains a vertical line must actually be a product of some closed subset of $X$ with $R$, it is clear that $\Gamma(X)=\{f \in \mathscr{F}$ : there exists some $x \in X$ with $f(x)$ finite $\}$. We show $\Gamma(X)$ is an open subset of $\left\langle\mathscr{F}, \tau_{a w}\right\rangle$, yielding its complete metrizability by Alexandrov's Theorem [27, §33].

Fix $f_{0} \in \Gamma(X)$ and $x_{0} \in X$ with $f_{0}\left(x_{0}\right)$ finite. By lower semicontinuity of $f_{0}$ there exist $\delta \in(0,1)$ such that for each $x \in x_{0}+3 \delta U$ we have $f_{0}(x)>$ $f_{0}\left(x_{0}\right)-1$. Consider the following bounded subset $B$ of $X \times R$ :

$$
B=\left(x_{0}+\delta U\right) \times\left[f_{0}\left(x_{0}\right)-4, f_{0}\left(x_{0}\right)-2\right] .
$$

Notice that if $(x, \alpha) \in B$ then $d\left((x, \alpha)\right.$, epi $\left.f_{0}\right)>\delta$. Let $C=B \cup\left\{\left(x_{0}, f\left(x_{0}\right)\right)\right\}$. Then

$$
\mathscr{A}=\left\{f \in \mathscr{F}: \sup _{(x, \alpha) \in C} \mid d((x, \alpha), \text { epi } f)-d\left((x, \alpha), \text { epi } f_{0}\right) \mid<\delta\right\}
$$

is a $\tau_{a w}$-neighborhood of $f_{0}$ in $\mathscr{F}$. We show that $\mathscr{A} \subset \Gamma(X)$. Fix $f \in \mathscr{A}$. Since $d\left(\left(x_{0}, f\left(x_{0}\right)\right)\right.$, epi $\left.f_{0}\right)=0$ we must have $d\left(\left(x_{0}, f\left(x_{0}\right)\right)\right.$, epi $\left.f\right)<\delta$. This means that there exists $(x, \alpha) \in$ epi $f$ with $x \in x_{0}+\delta U$ and $\left|\alpha-f\left(x_{0}\right)\right|<1$. Now $(x, \alpha-3) \in B$ so that

$$
d((x, \alpha-3) \text {, epi } f)>d\left((x, \alpha-3) \text {, epi } f_{0}\right)-\delta>0 \text {. }
$$

Thus $(x, \alpha-3) \notin$ epi $f$, and we have $\alpha-3<f(x) \leq \alpha$. Thus $f$ is somewhere finite and $f \in \Gamma(X)$.

Similar arguments show that $\left\langle\Gamma(X), \tau_{M}\right\rangle$ is completely metrizable when $X$ is reflexive and separable, for the function space will also be a $G_{\delta}$ subset of the completely metrizable space $\left\langle\mathscr{C}(X \times R), \tau_{M}\right\rangle$ [7, Theorem 4.3]. First, the set $\mathscr{F}=\{A \in \mathscr{C}(X \times R)$ : whenever $(x, \alpha) \in A$ and $\beta>\alpha$, then $(x, \beta) \in A\}$ is again easily shown to be $\tau_{M}$-closed in $\mathscr{C}(X \times R)$. Second, $\Gamma(X)$ is again an open subset of $\mathscr{F}$. To see this, fix $f_{0} \in \Gamma(X)$ and $x_{0} \in X$ with $f_{0}\left(x_{0}\right)$ finite. There exists $\alpha \in R$ with $\inf _{\left\|x-x_{0}\right\| \leq 1} f(x)>\alpha$ because $x_{0}+U$ is weakly compact and $f$ is weakly lower semicontinuous. Let $V$ be a norm open subset of $X \times R$ of diameter less than 1 containing $\left(x_{0}, f_{0}\left(x_{0}\right)\right)$ such that $V$ lies above $\left(x_{0}+U\right) \times\{\alpha\}$. Evidently,

$$
\mathscr{D} \equiv V^{-} \cap\left[\left(\left(x_{0}+U\right) \times\{\alpha\}\right)^{c}\right]^{+} \cap \mathscr{F}
$$


is a $\tau_{M}$-neighborhood of $f_{0}$ relative to $\mathscr{F}$, and if $f \in \mathscr{D}$, then there exists $x \in x_{0}+U$ with $\alpha<f(x)<f_{0}\left(x_{0}\right)+1$. Thus, $\mathscr{D} \subset \Gamma(X)$.

We anticipate that our next theorem will have numerous applications. An analagous result does hold for Mosco convergence, and the proof is much simpler (see, e.g., $[8,33])$.

Theorem 3.6. Let $X$ be a normed linear space. Suppose $\left\langle f_{n}\right\rangle$ is a sequence in $\Gamma(X)$ with $f=\tau_{a w}-\lim f_{n}$. Then for each $\alpha>v(f)$, we have $\operatorname{lev}(f ; \alpha)=$ $\tau_{a w}-\lim \operatorname{lev}\left(f_{n} ; \alpha\right)$.

Proof. Choose $\beta$ strictly between $\alpha$ and $v(f)$, and choose $\rho_{0}>|\alpha|$ such that for some $x_{0} \in \operatorname{int} \rho_{0} U$ we have $f\left(x_{0}\right)<\beta$. Since [int $\left.\rho_{0} U \times(-\infty, \beta)\right]^{-}$is a $\tau_{M}$-neighborhood of $f$, it is a $\tau_{a w}$-neighborhood of $f$. Thus, there exists $N_{1} \in Z^{+}$such that $n>N_{1}$ implies $f_{n} \in\left[\text { int } \rho_{0} U \times(-\infty, \beta)\right]^{-}$. Fix $\rho>\rho_{0}$ and $\varepsilon>0$; we produce $N \in Z^{+}$such that for each $n>N$, both of the following conditions hold:

(1) $\operatorname{lev}(f ; \alpha) \cap \rho U \subset \operatorname{lev}\left(f_{n} ; \alpha\right)+\varepsilon U$;

(2) $\operatorname{lev}\left(f_{n} ; \alpha\right) \cap \rho U \subset \operatorname{lev}(f ; \alpha)+\varepsilon U$.

Choose $\delta>0$ such that $\delta+2 \delta \rho /(\alpha-\beta+\delta)<\varepsilon$. Pick $N_{2} \in Z^{+}$so large that

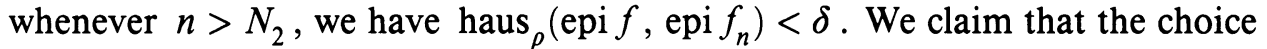
$N=N_{1}+N_{2}$ works. We verify that condition (1) holds; verification of (2) is exactly the same and is left to the reader.

Fix $n>N$ and $x \in \operatorname{lev}(f ; \alpha) \cap \rho U$. Since $\rho>|\alpha|$, we obtain $(x, \alpha) \in$ $\rho U \times[-\rho, \rho]$. Since $n>N_{2}$, there exists $\left(w_{n}, \alpha_{n}\right) \in$ epi $f_{n}$ with $\left\|w_{n}-x\right\|<\delta$ and $\left|\alpha_{n}-\alpha\right|<\delta$. Since $n>N_{1}$ and $\rho>\rho_{0}$, there exists $z_{n} \in \rho U$ with $f_{n}\left(z_{n}\right)<\beta$. Let $\lambda=(\alpha-\beta) /(\alpha-\beta+\delta)$; we will show

(i) $\lambda w_{n}+(1-\lambda) z_{n} \in \operatorname{lev}\left(f_{n} ; \alpha\right)$;

(ii) $\left\|\lambda w_{n}+(1-\lambda) z_{n}-x\right\| \leq \varepsilon$.

Condition (i) follows easily from $f_{n}\left(w_{n}\right) \leq \alpha_{n}<\alpha+\delta$ and $f_{n}\left(z_{n}\right)<\beta$ :

$$
\begin{aligned}
f_{n}\left(\lambda w_{n}+(1-\lambda) z_{n}\right) & \leq \lambda f_{n}\left(w_{n}\right)+(1-\lambda) f_{n}\left(z_{n}\right) \\
& <\lambda(\alpha+\delta)+(1-\lambda) \beta \\
& =\frac{(\alpha-\beta)(\alpha+\delta)+\delta \beta}{(\alpha-\beta+\delta)}=\alpha .
\end{aligned}
$$

Condition (ii) is immediate from the choice of $\delta$ and the fact that both $z_{n}$ and $x$ lie in $\rho U$ :

$$
\begin{aligned}
\left\|\lambda w_{n}+(1-\lambda) z_{n}-x\right\| & \leq \lambda\left\|w_{n}-x\right\|+(1-\lambda)\left\|z_{n}-x\right\| \\
& <\left\|w_{n}-x\right\|+\left(\frac{\delta}{\alpha-\beta+\delta}\right)\left\|z_{n}-x\right\| \\
& <\delta+2 \delta \rho /(\alpha-\beta+\delta)<\varepsilon .
\end{aligned}
$$

This completes the proof of $\tau_{a w}$-convergence of sublevel sets at a fixed height above $v(f)$. 
It is easy to see that $\tau_{a w}$-convergence of $\left\langle f_{n}\right\rangle$ to $f$ does not guarantee that $\operatorname{lev}(f ; v(f))=\tau_{a w}-\lim \operatorname{lev}\left(f_{n} ; v(f)\right):$ take $f_{n}(x) \equiv 1 / n$ and $f(x) \equiv 0$. As a first application of Theorem 3.6, we have

Theorem 3.7. Let $X$ be a normed linear space, and let $f, f_{1}, f_{2}, f_{3}, \ldots$ be functions in $\Gamma(X)$ with $f=\tau_{a w}-\lim f_{n}$. Then

(a) $v(f) \geq \lim \sup _{n \rightarrow \infty} v\left(f_{n}\right)$;

(b) if for some $\alpha>v(f), \operatorname{lev}(f ; \alpha)$ is bounded, then $v(f)=\lim _{n \rightarrow \infty} v\left(f_{n}\right)$.

Proof. (a) Upper semicontinuity of the value function holds for the weaker Mosco topology; in fact, it holds for the topology generated by all sets of the form $\{f \in \Gamma(X)$ : epi $f \cap V \neq \varnothing\}=\Gamma(X) \cap V^{-}$where $V$ is norm open in $X \times R$ (see, e.g., [2, p. 128]).

(b) Clearly, $v(f) \leq \liminf _{n \rightarrow \infty} v\left(f_{n}\right)$ holds if $v(f)=-\infty$. Suppose now that $v(f)$ is finite and for some $\varepsilon>0$, we have $v\left(f_{n}\right)<v(f)-3 \varepsilon$ for each $n$ in some infinite subset $J$ of $Z^{+}$. For each $n \in J$, we may choose $c_{n} \in X$ with $f_{n}\left(c_{n}\right)<v(f)-2 \varepsilon$. By Lemma 3.1 and Theorem 3.6, there exists $N_{1} \in Z^{+}$ and $\rho>|v(f)|+3 \varepsilon$ such that $\operatorname{lev}\left(f_{n} ; v(f)-2 \varepsilon\right) \subset \rho U$ for each $n>N_{1}$. Pick $N_{2}>N_{1}$ such that for each $n>N_{2}$ we have haus ${ }_{\rho}$ (epi $f$, epi $\left.f_{n}\right)<\varepsilon$. Fix $n \in J$ with $n>N_{2}$. Now

$$
\left(c_{n}, v(f)-2 \varepsilon\right) \in \operatorname{epi} f_{n} \cap \rho U \times[-\rho, \rho] ;
$$

so, there exists $(x, \alpha) \in$ epi $f$ with $\left\|(x, \alpha)-\left(c_{n}, v(f)-2 \varepsilon\right)\right\|<\varepsilon$. This means that $f(x) \leq \alpha<v(f)-\varepsilon$, a contradiction. We conclude that in this case, we also have $v(f) \leq \liminf _{n \rightarrow \infty} v\left(f_{n}\right)$.

Example. Part (b) of Theorem 3.7 fails for Mosco convergence, even in $l_{2}$. To see this, let $f$ be the indicator function for the origin (a well-posed function), and for each $n$, let $f_{n}: l_{2} \rightarrow R$ be defined by

$$
f_{n}(x)=\left\{\begin{array}{l}
\max \{-\alpha / n,-1\}, \quad \text { if } x=\alpha e_{n} \text { and } \alpha \geq 0 ; \\
\infty, \quad \text { otherwise. }
\end{array}\right.
$$

Obviously, $v(f)=0$ and $v\left(f_{n}\right)=-1$ for each $n$. Using the fact that the origin is the weak limit of $\left\langle e_{n}\right\rangle$, it is easy to see that $f=\tau_{M}-\lim f_{n}$.

We refer the reader to [5] for a sharper version of Theorem 3.7(b). The converse of Theorem 3.6 fails: for any $X$ and any $A \in \mathscr{C}(X)$, let $f_{n}=I(\cdot, A)-$ $n$ and let $f=I(\cdot, A)$. We do, however, have a partial converse for Theorem 3.6 , which holds without any convexity assumptions whatsoever.

Theorem 3.8. Let $X$ be a normed linear space and let $f, f_{1}, f_{2}, \ldots$ be (convex) functions on $X$ satisfying $\operatorname{lev}(f ; \alpha)=\tau_{a w}-\lim \operatorname{lev}\left(f_{n} ; \alpha\right)$ for each $\alpha>v(f)$. Then if $v(f) \leq \liminf _{n \rightarrow \infty} v\left(f_{n}\right)$, we have $f=\tau_{a w}-\lim f_{n}$.

Proof. Let $\rho_{0}$ be a positive scalar exceeding $v(f)$. Fix $\rho>\rho_{0}$ and $\varepsilon>0$. We produce $N \in Z^{+}$such that for each $n>N$, haus ${ }_{\rho}\left(f, f_{n}\right) \leq \varepsilon$. 
Choose $k \in Z^{+}$with $4 \rho / k<\varepsilon$. Also, choose $N_{1} \in Z^{+}$such that for each $n>N_{1}$ we have $v\left(f_{n}\right) \geq v(f)-2 \rho / k$. Write $\alpha_{j}=-\rho+j(2 \rho / k)$ for each $j \in\{1,2,3, \ldots, k+1\}$, and let $j_{0}$ be the smallest index in $\{1,2,3, \ldots, k\}$ with $\alpha_{j_{0}}>v(f) \quad\left(j_{0}\right.$ exists because $\left.\rho>\rho_{0}>v(f)\right)$. By our assumption on convergence of sublevel sets, there exists $N_{2} \in Z^{+}$such that haus ${ }_{\rho}\left(\operatorname{lev}\left(f ; \alpha_{j}\right)\right.$, $\left.\operatorname{lev}\left(f_{n} ; \alpha_{j}\right)\right)<\varepsilon$ for each $n>N_{2}$ and for each $j$ with $j_{0} \leq j \leq k+1$. Fix $n>N \equiv N_{1}+N_{2}$. We verify both of the following:

(i) $e$ (epi $f \cap \rho U \times[-\rho, \rho]$, epi $\left.f_{n}\right) \leq \varepsilon$, and

(ii) $e\left(\right.$ epi $f_{n} \cap \rho U \times[-\rho, \rho]$, epi $\left.f\right) \leq \varepsilon$.

The cases are not entirely symmetric, (ii) being the more subtle. For (i), fix $(x, \alpha) \in$ epi $f \cap \rho U \times[-\rho, \rho]$. Let $j \in\{1,2, \ldots, k+1\}$ be the minimal index with $\alpha<\alpha_{j}$. Then $x \in \operatorname{lev}\left(f ; \alpha_{j}\right)$, and since $n>N_{2}$, there exists $z_{n} \in \operatorname{lev}\left(f_{n} ; \alpha_{j}\right)$ with $\left\|z_{n}-x\right\|<\varepsilon$. Since $\alpha_{j}-\varepsilon<\alpha_{j}-2 \rho / k \leq \alpha<\alpha_{j}$, we have $\left\|\left(z_{n}, \alpha_{j}\right)-(x, \alpha)\right\|<\varepsilon$, and (i) follows.

To establish (ii), fix $\left(x_{n}, \beta_{n}\right) \in \operatorname{epi} f_{n} \cap \rho U \times[-\rho, \rho]$. In this case, let $j \in\{1,2, \ldots, k\}$ be the minimal index with $\beta_{n} \leq \alpha_{j}$. Since $n>N_{1}$ and $j \leq k$, we have

$$
v(f) \leq v\left(f_{n}\right)+2 \rho / k \leq \beta_{n}+2 \rho / k \leq \alpha_{j}+2 \rho / k=\alpha_{j+1} \leq \alpha_{k+1} .
$$

In particular, $j_{0} \leq j+1 \leq k+1$. Since $x_{n} \in \operatorname{lev}\left(f_{n} ; \alpha_{j+1}\right)$ and $n>N_{2}$, there exists $z \in \operatorname{lev}\left(f ; \alpha_{j+1}\right)$ with $\left\|z-x_{n}\right\|<\varepsilon$. Then $\left(z, \alpha_{j+1}\right) \in \operatorname{epi} f$, and

$$
\begin{aligned}
\left\|\left(z, \alpha_{j+1}\right)-\left(x_{n}, \beta_{n}\right)\right\| & =\max \left\{\left\|z-x_{n}\right\|,\left|\alpha_{j+1}-\beta_{n}\right|\right\} \\
& <\max \{\varepsilon, 4 \rho / k\}=\varepsilon .
\end{aligned}
$$

This establishes (ii), completing the proof.

We note that the condition $\liminf _{n \rightarrow \infty} v\left(f_{n}\right) \geq v(f)$ in Theorem 3.8 is really no weaker than the condition $v(f)=\lim _{n \rightarrow \infty} v\left(f_{n}\right)$, by virtue of $\tau_{a w}{ }^{-}$ convergence of sublevel sets above height $v(f)$.

\section{WELL-POSEDNESS OF CONVEX FUNCTIONS}

The main results of this section depend on the following characterization of well-posedness, due to Furi and Vignoli: a proper lower semicontinuous function on a complete metric space is well-posed if and only if $\inf \{\operatorname{diam} \operatorname{lev}(f ; \alpha)$ : $\alpha>v(f)\}=0[20]$. This has been used frequently in more classical settings (see, e.g., $[17,29,34])$.

Theorem 4.1. Let $X$ be a Banach space. Then $f \in \Gamma(X)$ is well-posed if and only if whenever $f=\tau_{a w}-\lim f_{n}$ and $x_{n} \in \operatorname{iev}\left(f_{n} ; v\left(f_{n}\right)+1 / n\right)$ for each $n$, then $\left\langle x_{n}\right\rangle$ is convergent (to the unique minimizer of $f$ ).

Proof. For sufficiency, simply take $f_{n}=f$ for each $n$. For necessity, let $x_{0}$ be the unique minimizer of $f$, let $\left\langle f_{n}\right\rangle$ and $\left\langle x_{n}\right\rangle$ be as described above, and let $\varepsilon>0$. By the Furi-Vignoli characterization of well-posedness, there exists $\delta>0$ such that $\operatorname{diam} \operatorname{lev}(f ; v(f)+\delta)<\varepsilon / 2$. By Lemmas 3.1 and Theorem 
3.6, $\left\langle\operatorname{lev}\left(f_{n} ; v(f)+\delta\right)\right\rangle$ is actually convergent to $\operatorname{lev}(f ; v(f)+\delta)$ in Hausdorff distance. By upper semicontinuity of the value function there exists $N \in Z^{+}$ such that $1 / N<\delta / 2$ and for each $n>N$ both $v\left(f_{n}\right)<v(f)+\delta / 2$ and haus $\left(\operatorname{lev}\left(f_{n} ; v(f)+\delta\right), \operatorname{lev}(f ; v(f)+\delta)\right)<\varepsilon / 2$. As a result, for each $n>N$ we have $\left\|x_{n}-x_{0}\right\|<\varepsilon$.

Immediate consequences of Theorem 3.7 and Theorem 4.1 are these: if $f$ is well-posed, then $\tau_{a w}$-convergence of $\left\langle f_{n}\right\rangle$ to $f$ drives minimizers of $\left\langle f_{n}\right\rangle$ (if they exist) into the unique minimizer of $f$, and $\lim _{n \rightarrow \infty} v\left(f_{n}\right)=v(f)$. Theorem 4.1 fails for Mosco convergence in $l_{2}$. In fact one can construct a sequence $\left\langle f_{n}\right\rangle$ in $\Gamma\left(l_{2}\right)$ Mosco convergent to $f(x)=\|x\|^{2}$ such that for each $n, e_{n} \in \arg \min f_{n}$ (see [28, Example 4.9]).

There is a generalization of Theorem 4.1 which we will record without proof. Recall that a proper lower semicontinuous function $f$ is well-posed in the generalized sense (g.w.p.) [28] provided each minimizing sequence for $f$ contains a convergent subsequence. Easily, it is seen that $f$ is g.w.p. if and only if $\arg \min f$ is nonempty and compact, and

$$
\inf _{\alpha>v(f)} e(\operatorname{lev}(f ; \alpha), \arg \min f)=0 .
$$

Following the proof of Theorem 4.1, we obtain

Theorem 4.2. Let $X$ be a Banach space. Then $f \in \Gamma(X)$ is well-posed in the generalized sense if and only if whenever $f=\tau_{a w}-\lim f_{n}$ and for each $n$, $x_{n} \in \operatorname{lev}\left(f_{n} ; v\left(f_{n}\right)+\varepsilon_{n}\right)$ where $\left\langle\varepsilon_{n}\right\rangle \rightarrow 0$, then $\left\langle x_{n}\right\rangle$ has a convergent subsequence (to a minimizer of $f$ ).

Recall that a $G_{\delta}$ subset of a topological space is one that can be expressed as an intersection of a countable family of open sets. Now let $X$ be an arbitrary Banach space. We intend to show that the well-posed functions form a dense and $G_{\delta}$ subset of the function space $\left\langle\Gamma(X), \tau_{a w}\right\rangle$. This result was first obtained in finite dimensions by Lucchetti and Patrone [30]; for refinements, the reader may consult $[13,34]$. By the Baire category theorem, a dense and $G_{\delta}$ subset of a complete metric space is most of the space; so, we will be entitled to say most functions in $\left\langle\Gamma(X), \tau_{a w}\right\rangle$ are well-posed, by virture of Lemma 3.5.

We obtain $\tau_{a w}$-density of the well-posed problems in $\Gamma(X)$ rather routinely through successive approximations.

Lemma 4.3. Let $X$ be a normed linear space. Suppose $f \in \Gamma(X)$ and $\arg \min f$ $\neq \varnothing$. Then there exists a sequence $\left\langle f_{n}\right\rangle$ of well-posed functions in $\Gamma(X) \tau_{a w}$ convergent to $f$.

Proof. Fix $x_{0} \in \arg \min f$, and for each $n \in Z^{+}$, let $f_{n} \in \Gamma(X)$ be defined by

$$
f_{n}(x)=f(x)+\left\|x-x_{0}\right\| / n .
$$

Evidently, each $f_{n}$ is well-posed (with unique minimum at $x_{0}$ ).

Choose $\rho_{0}$ with $\left\|x_{0}\right\|<\rho_{0}$. Fix $\rho>\rho_{0}$. Since epi $f_{n} \subset$ epi $f$, we have $e\left(\right.$ epi $f_{n} \cap \rho U \times[-\rho, \rho]$, epi $\left.f\right)=0$. On the other hand, if $(x, \alpha) \in \operatorname{epi} f$ where 
$\|x\| \leq \rho$ and $|\alpha| \leq \rho$, then $\left\|x-x_{0}\right\| \leq 2 \rho$ so that $(x, \alpha+2 \rho / n) \in$ epi $f_{n}$. Thus, $e\left(\right.$ epi $f \cap \rho U \times[-\rho, \rho]$, epi $\left.f_{n}\right) \leq 2 \rho / n$. As a result, haus ${ }_{\rho}$ (epi $f$, epi $\left.f_{n}\right) \leq$ $2 \rho / n$, and $f=\tau_{a w}-\lim f_{n}$.

Lemma 4.4. Let $X$ be a normed linear space. Then the well-posed functions are dense in $\left\langle\Gamma(X), \tau_{a w}\right\rangle$.

Proof. By Lemma 4.3, it suffices to show that the functions with nonempty $\arg \min$ are dense in $\Gamma(X)$. For each $f \in \Gamma(X)$ with $\arg \min f=\varnothing$, we produce a sequence $\left\langle f_{n}\right\rangle$ in $\Gamma(X)$ that is $\tau_{a w}$-convergent to $f$ with $\arg \min f_{n} \neq$ $\varnothing$ for each $n$.

Suppose first that $f$ is unbounded below. For each $n$, let

$$
f_{n}(x)=\max \{f(x),-n\} .
$$

Evidently, $\arg \min f_{n}=\{x: f(x) \leq-n\}$, a nonempty set, and by Lemma 3.3, $f=\tau_{a w}-\lim f_{n}$. If $f$ is bounded below but has no minimum value, for each $n \in Z^{+}$, pick $x_{n} \in X$ with $v(f)<f\left(x_{n}\right)<v(f)+1 / n$. For each $n$ let $f_{n}$ be the lower semicontinuous convex function whose epigraph is the closure of the convex hull of $\left(x_{n}, v(f)\right)$ and epi $f$. It is routine to check that haus(epi $f$, epi $\left.f_{n}\right)<1 / n$, and since the Hausdorff metric topology is stronger than the epi-distance topology, we have $f=\tau_{a w}-\lim f_{n}$.

To show that the well-posed functions form a $G_{\delta}$-subset of the function space, we apply Lemma 3.2 and Theorem 3.6.

Theorem 4.5. Let $X$ be a Banach space. If $\Gamma(X)$ is equipped with the epidistance topology $\tau_{a w}$, then in the sense of Baire category, most elements of $\Gamma(X)$ are well-posed.

Proof. By Lemma 3.5, the function space is a Baire space; so the statement most elements of $\Gamma(X)$ are well-posed at least makes sense. By Lemma 4.4, the well-posed functions are dense in the function space. Since $X$ is complete, by our remarks at the beginning of this section, $f \in \Gamma(X)$ is well-posed if and only if for each $i \in Z^{+}$there exists $\alpha>v(f)$ with $\operatorname{diam} \operatorname{lev}(f ; \alpha)<1 / i$. Thus, $f$ is not well-posed if and only if $f \in \mathscr{F}_{i}$ for some $i \in Z^{+}$, where

$$
\mathscr{F}_{i}=\{f \in \Gamma(X) \text { : for each } \alpha>v(f) \text {, we have } \operatorname{diam} \operatorname{lev}(f ; \alpha) \geq 1 / i\} .
$$

It remains only to show that each $\mathscr{F}_{i}$ is $\tau_{a w}$-closed. To this end, let $\left\langle f_{n}\right\rangle$ be a sequence in $\mathscr{F}_{i} \tau_{a w}$-convergent to $f$, and let $\alpha>v(f)$ be arbitrary. By Theorem 3.6, $\operatorname{lev}(f ; \alpha)=\tau_{a w}-\lim \operatorname{lev}\left(f_{n} ; \alpha\right)$; so, by Lemma 3.2 and Theorem 3.7(a), we have

$$
\operatorname{diam} \operatorname{lev}(f ; \alpha)=\lim _{n \rightarrow \infty} \operatorname{diam} \operatorname{lev}\left(f_{n} ; \alpha\right) \geq 1 / i .
$$

We conclude that $f \in \mathscr{F}_{i}$, completing the proof.

We note that Theorem 4.5 has a dual interpretation. It is well-known that $f \rightarrow f^{*}$ is a bijection from $\Gamma(X)$ to $\Gamma^{*}\left(X^{*}\right)$, where $\Gamma^{*}\left(X^{*}\right)$ denotes the proper, 
weak ${ }^{*}$-lower semicontinuous, convex functions on $X^{*}[24, \S 14]$. Since the conjugate map is a homeomorphism of $\left\langle\Gamma(X), \tau_{a w}\right\rangle$ onto $\left\langle\Gamma^{*}\left(X^{*}\right), \tau_{a w}\right\rangle$ for an arbitrary normed linear space [10], it follows from Lemma 3.5 that $\left\langle\Gamma^{*}\left(X^{*}\right), \tau_{a w}\right\rangle$ is completely metrizable when $X$ is a Banach space. Now by the celebrated Asplund-Rockafellar Theorem [1], for any Banach space $X, f \in \Gamma(X)$ is wellposed if and only if $f^{*}$ is Frechet differentiable at the origin. Putting this all together, we obtain

Theorem 4.6. Let $X$ be a Banach space, and let $\Gamma^{*}\left(X^{*}\right)$ be the proper, weak ${ }^{*}$ lower semicontinuous, convex functions on $X^{*}$. Then $\left\langle\Gamma^{*}\left(X^{*}\right), \tau_{a w}\right\rangle$ is completely metrizable, and most functions in $\left\langle\Gamma^{*}\left(X^{*}\right), \tau_{a w}\right\rangle$ are Frechet differentiable at the origin.

If $X$ is any normed linear space, then the nonconstant continuous affine functionals on $X$ are open in the Hausdorff metric topology on $\Gamma(X)$. Thus, the well-posed functions cannot even be dense in $\Gamma(X)$ so topologized. Now let $X$ be any infinite dimensional normed linear space. We intend to show that with respect to the Mosco topology on $\Gamma(X)$, the set of functions that are unbounded below forms a dense and $G_{\delta}$ subset of $\Gamma(X)$. We require Lemma 2.2 of [8], which we reproduce for the convenience of the reader (see also [2, $\S 3.5 .2])$.

Lemma 4.7. Let $X$ be a normed linear space, and let $K$ be a weakly compact subset of $X \times R$. Suppose $f \in \Gamma(X) \cap\left(K^{c}\right)^{+}$. Then there exists $\varepsilon>0$ and $a$ finite collection of pairs $\left(y_{1}, \mu_{1}\right),\left(y_{2}, \mu_{2}\right), \ldots,\left(y_{m}, \mu_{m}\right)$ in $X^{*} \times R$ such that $u(\cdot) \equiv \sup _{k \leq m}\left\langle\cdot, y_{k}\right\rangle-\mu_{k} \in\left(K^{c}\right)^{+}$, and for each $k \leq m$, we have

$$
\inf _{x \in X}\left[f(x)-\left\langle x, y_{k}\right\rangle+\mu_{k}\right]>\varepsilon .
$$

Our construction rests on the next elementary fact.

Lemma 4.8. Let $X$ be a normed linear space, and let $\left\{y_{1}, y_{2}, \ldots, y_{m}\right\}$ be vectors in $X^{*}$. The following are equivalent:

(1) $\theta^{*} \notin \operatorname{conv}\left\{y_{1}, y_{2}, \ldots, y_{m}\right\}$;

(2) there exists $x_{0} \in X$ such that for each $k \leq m$, we have $\left\langle x_{0}, y_{k}\right\rangle>0$.

Proof. (1) $\Rightarrow(2)$. Let $P=\operatorname{conv}\left\{y_{1}, y_{2}, \ldots, y_{m}\right\}$; since $P$ is weak ${ }^{*}$-compact, by the separation theorem applied to $\theta^{*}$ and $P$, there exists $x_{0} \in X$ such that $\left\langle x_{0}, \theta^{*}\right\rangle<\inf _{y \in P}\left\langle x_{0}, y\right\rangle$. In particular, $\left\langle x_{0}, y_{k}\right\rangle$ is positive for each index $k$.

(2) $\Rightarrow(1)$. Suppose (1) fails. If some $y_{k}$ is the origin of $X^{*}$, then clearly (2) fails. Otherwise, there exist nonnegative scalars $\alpha_{1}, \ldots, \alpha_{m}$ summing to 1 at least two of which are positive such that $\alpha_{1} y_{1}+\alpha_{2} y_{2}+\cdots+\alpha_{m} y_{m}=\theta^{*}$. Without loss of generality, we may assume $\alpha_{1} \neq 0$. Clearly, whenever $x \in X$ satisfies $\left\langle x, y_{k}\right\rangle>0$ for $k=2,3, \ldots, m$, we must have $\left\langle x, y_{1}\right\rangle<0$, because $y_{1}=\left(-1 / \alpha_{1}\right)\left(\alpha_{2} y_{2}+\cdots+\alpha_{m} y_{m}\right)$, and for some $k \geq 2$, the coefficient $\alpha_{k}$ is positive. Thus, (2) again fails. 
Theorem 4.9. Let $X$ be an infinite dimensional normed linear space. Then $\mathscr{D} \equiv$ $\{f \in \Gamma(X): f$ is unbounded below $\}$ is a dense and $G_{\delta}$ subset of $\left\langle\Gamma(X), \tau_{M}\right\rangle$.

Proof. Evidently, $\mathscr{D}=\bigcap_{n \in Z^{+}}\left(\Gamma(X) \cap(X \times(-\infty,-n))^{-}\right)$; so, $\mathscr{D}$ is a $G_{\delta}$ subset of the function space. Only the density of $\mathscr{D}$ is in question. To establish density, let $\mathscr{U} \equiv V_{1}^{-} \cap V_{2}^{-} \cap \cdots \cap V_{n}^{-} \cap\left(K^{c}\right)^{+}$be a basic $\tau_{M}$-neighborhood of a proper, convex, lower semicontinuous function $f$ on $X$. For each $i \leq n$, choose $\left(x_{i}, \alpha_{i}\right) \in$ epi $f \cap V_{i}$. Let $\left\{\left(y_{k}, \mu_{k}\right): 1 \leq k \leq m\right\}$ be the points in $X^{*} \times R$ whose existence is guaranteed by Lemma 4.7 with respect to the function $f$ and the weakly compact set $K$. For each $k \leq m$, write $\beta_{k}=\mu_{k}-\varepsilon / 2$, and let $h: X \rightarrow R$ be defined by

$$
h(x)=\sup _{1 \leq k \leq m}\left\langle x, y_{k}\right\rangle-\beta_{k} .
$$

Lemma 4.7 says that for each $(x, \alpha) \in K$, we have $\alpha<h(x)-\varepsilon / 2$, and for each $(x, \alpha) \in$ epi $f$, we have $\alpha>h(x)+\varepsilon / 2$ (in particular, $\alpha_{i}>h\left(x_{i}\right)+\varepsilon / 2$ for $i=1,2, \ldots, n)$.

Next, let $K_{0}$ be the projection of $K$ onto $X$, and choose $\lambda>0$ such that $K_{0} \cup\left\{x_{1}, x_{2}, \ldots, x_{n}\right\} \subset \lambda U$. Since $X$ is infinite dimensional, there exist linearly independent vectors $z_{1}, z_{2}, \ldots, z_{m}$ in $X^{*}$ such that $\left\|z_{k}-y_{k}\right\|<\varepsilon / 2 \lambda$ for each index $k \leq m$. Consider the convex function $g: X \rightarrow R$ defined by

$$
g(x)=\sup _{1 \leq k \leq m}\left\langle x, z_{k}\right\rangle-\beta_{k} .
$$

By the choice of $\lambda$ and the definition of $h$, it is clear that epi $g \cap K=\varnothing$ and that $\alpha_{i}>g\left(x_{i}\right)$ for $i=1, \ldots, n$, so that $\left(x_{i}, \alpha_{i}\right) \in$ epi $g$ for each $i$. This means that $g \in \mathscr{U}$. Invoking Lemma 4.8, there exists $x_{0} \in X$ such that for each $k \leq m$, we have $\left\langle x_{0}, z_{k}\right\rangle>0$. As a result, for each $k \leq m$, we have $\lim _{\alpha \rightarrow-\infty}\left\langle\alpha x_{0}, z_{k}\right\rangle-\beta_{k}=-\infty$, so that $g$ fails to be bounded below when restricted to the ray $\left\{\alpha x_{0}: \alpha \leq 0\right\}$. This proves that the functions unbounded below are $\tau_{M}$-dense in $\Gamma(X)$.

\section{Applications to metric PROJECTIONS}

Let $A \in \mathscr{C}(X)$ and $x \in X$ be arbitrary. Recall $[23,24]$ that the metric projection of $x$ onto $A$ is the possibly empty convex set

$$
P(x, A)=\{a \in A:\|x-a\|=d(x, A)\} .
$$

The convex set $A$ is called Chebyshev provided $P(x, A)$ is a singleton for each $x \in X$. If $X$ is reflexive then $P(x, A)$ is nonempty for each $x$ and $A$; in fact, this property characterizes reflexivity. Are most elements of $\left\langle\mathscr{C}(X), \tau_{a w}\right\rangle$ Chebyshev in a reflexive space? The answer is affirmative!

Recall that a convex set is called a convex body provided its interior is nonempty, and rotund provided its boundary contains no line segments. By a well-known theorem of Klee [26], within the space of closed and bounded convex bodies topologized by Hausdorff distance, the sets that are rotund form a dense and $G_{\delta}$ subset. Each such set is obviously Chebyshev. 
Theorem 5.1. Let $X$ be a reflexive Banach space. Then most elements of $\langle\mathscr{C}(X)$, $\left.\tau_{a w}\right\rangle$ are Chebyshev.

Proof. Let $\mathscr{C}_{0}(X)$ denote the closed and bounded convex bodies in $X$. By Lemma 3.1, $\tau_{a w}$ reduces to the Hausdorff metric topology on $\mathscr{C}_{0}(X)$. Thus, by Klee's Theorem, it suffices to show that $\mathscr{E}_{0}(X)$ is dense and open in $\left\langle\mathscr{C}(X), \tau_{a w}\right\rangle$. Density is easy to see, for if $A \in \mathscr{C}(X)$, then $A=\tau_{a w}-\lim (A+(1 / n) U)$, and by Lemma 3.3, for each $n \in Z^{+}, A+(1 / n) U=\tau_{a w}-\lim _{k \rightarrow \infty} k U \cap(A+(1 / n) U)$. By Lemma 3.4 the closed convex bodies are open in $\left\langle\mathscr{C}(X), \tau_{a w}\right\rangle$, and by Lemma 3.1 the bounded elements of $\mathscr{E}(X)$ are open in $\left\langle\mathscr{C}(X), \tau_{a w}\right\rangle$. Thus, $\mathscr{C}_{0}(X)$ is dense and open in $\left\langle\mathscr{C}(X), \tau_{a w}\right\rangle$, concluding the proof.

This general line of reasoning was used in [11] to establish Theorem 5.1 in finite dimensions, before the author was aware of $\tau_{a w}$. Theorem 5.1 fails with respect to the box norm in finite dimensions if $\tau_{a w}$ is replaced by the Hausdorff metric topology [17]. It is not known whether Theorem 5.1 holds even in a separable reflexive space if $\tau_{a w}$ is replaced by $\tau_{M}$ (but see Theorem 5.4 of [7]). On the negative side, $\mathscr{C}_{0}(X)$ is a set of first category in $\left\langle\mathscr{C}(X), \tau_{M}\right\rangle$ whenever $X$ is infinite dimensional [7, p. 252].

For each $x \in X$ let $\varphi_{x}: X \rightarrow R$ be defined by $\varphi_{x}(z)=\|x-z\|$. For each $A \in \mathscr{C}(X)$, we clearly have $\arg \min \varphi_{x}+I(\cdot, A)=P(x, A)$. We intend to show that for each $x \in A, \varphi_{x}+I(\cdot, A)$ is well-posed for most $A \in \mathscr{C}(X)$, provided $X$ is reflexive.

Lemma 5.2. Let $X$ be a normed linear space. Suppose $\left\langle x_{n}\right\rangle \rightarrow x$ and $A=$ $\tau_{a w}-\lim A_{n}$. Then $\varphi_{x}+I(\cdot, A)=\tau_{a w}-\lim \varphi_{x_{n}}+I\left(\cdot, A_{n}\right)$.

Proof. Let $\varepsilon>0, \rho>0$. Choose $N$ so large that $n>N$ implies both $\left\|x_{n}-x\right\|<\varepsilon / 2$ and haus ${ }_{\rho}\left(A_{n}, A\right)<\varepsilon / 2$. Fix $n>N$. We must show that both of the following conditions hold:

(i) $e\left(\operatorname{epi} \varphi_{x_{n}}+I\left(\cdot, A_{n}\right) \cap \rho U \times[-\rho, \rho]\right.$, epi $\left.\varphi_{x}+I(\cdot, A)\right) \leq \varepsilon$;

(ii) $e\left(\right.$ epi $\varphi_{x}+I(\cdot, A) \cap \rho U \times[-\rho, \rho]$, epi $\left.\varphi_{x_{n}}+I\left(\cdot, A_{n}\right)\right) \leq \varepsilon$.

We only establish (i), as the proofs are identical. Fix $a_{n} \in A_{n} \cap \rho U$ and $\alpha \geq$ $\left\|a_{n}-x_{n}\right\|$ (we need not assume that $\alpha \in[-\rho, \rho]$ ). Since haus ${ }_{\rho}\left(A, A_{n}\right)<\varepsilon / 2$, there exists $c_{n} \in A$ with $\left\|c_{n}-a_{n}\right\|<\varepsilon / 2$. Clearly,

$$
\varphi_{x}\left(c_{n}\right)=\left\|c_{n}-x\right\|<\left\|a_{n}-x_{n}\right\|+\varepsilon=\varphi_{x_{n}}\left(a_{n}\right)+\varepsilon \leq \alpha+\varepsilon .
$$

Thus, $\left(c_{n}, \alpha+\varepsilon\right) \in \operatorname{epi} \varphi_{x}+I(\cdot, A)$, and $\left\|\left(a_{n}, \alpha\right)-\left(c_{n}, \alpha+\varepsilon\right)\right\| \leq \varepsilon$.

In the literature a closed convex set $A$ is called approximatively compact [19, 24] provided for each $x \in X$ the convex function $\varphi_{x}+I(\cdot, A)$ is well-posed in the generalized sense. Of course, this yields compactness of $P(x, A)$ for each $x$. By Theorem 4.2 and Lemma 5.2, approximative compactness for a convex set $A$ in a Banach space may be characterized by this stronger property: at each $x \in X$, whenever $\left\langle x_{n}\right\rangle \rightarrow x, A=\tau_{a w}-\lim A_{n}$, and $\left\langle d\left(x, a_{n}\right)\right\rangle \rightarrow d(x, A)$ where for each $n, a_{n} \in A_{n}$, then $\left\langle a_{n}\right\rangle$ has a subsequence convergent to a point of $P(x, A)$. 
It is worthwhile saying what approximative compactness yields for the metric projection, viewed as a bivariate set valued function, in the language of multifunctions. Recall that a set valued function $F$ from a topological space $T$ to a topological space $Y$ is called upper semicontinuous [27, $\S 18]$ at $t_{0} \in T$ provided whenever $V$ is a neighborhood of $F\left(t_{0}\right)$, there exists a neighborhood $W$ of $t_{0}$ such that for each $t \in W$ we have $F(t) \subset V$ (in the notation of $\S 2$, for each neighborhood $V$ of $F\left(t_{0}\right),\left\{t: F(t) \in V^{+}\right\}$contains a neighborhood of $\left.t_{0}\right)$. By the remarks above, $(x, A) \rightarrow P(x, A)$ is upper semicontinuous at each $(x, A)$ where $A$ is approximatively compact, provided we equip $\mathscr{C}(X)$ with $\tau_{a w}$. It is known [7] that for reflexive $X$, the metric projection is weakly upper semicontinuous at each point of $X \times \mathscr{C}(X)$, provided $\mathscr{C}(X)$ is equipped with $\tau_{M}$. This of course remains true if we equip $\mathscr{C}(X)$ with the stronger topology $\tau_{a w}$.

Lemma 5.3. Let $X$ be a reflexive Banach space. Then for each $x \in X,\{A \in$ $\mathscr{C}(X): \varphi_{x}+I(\cdot, A)$ is well-posed $\}$ is dense in $\mathscr{C}(X)$.

Proof. Without loss of generality, we may assume $x=\theta$. If $\theta \in A$, then $\varphi_{\theta}+$ $I(\cdot, A)$ is already well-posed. Otherwise, by Lemma 3.3, it suffices to assume $A$ is weakly compact, because $A$ can be $\tau_{a w}$-approximated by $\langle A \cap n U\rangle$. Under this assumption, choose $a_{0} \in A$ nearest $\theta$. Obviously, $\left\langle\operatorname{conv}\left(\left\{(1-1 / n) a_{0}\right\} \cup A\right)\right\rangle$ $\tau_{a w}$-approximates $A$; so, it suffices to show that $\varphi_{\theta}+I\left(\cdot, \operatorname{conv}\left(\left\{\alpha a_{0}\right\} \cup A\right)\right)$ is well-posed for each $\alpha \in(0,1)$.

To see this, we draw on a construction presented in Lemma 5.2 of [7]. Fix $\alpha \in(0,1)$, choose $\rho$ such that $A \subset \rho U$, and fix $\lambda>0$. In order to prove well-posedness, it suffices to show that there exists $\mu>0$ depending only on $\lambda$, $\alpha$, and $\rho$ such that whenever $z \in \operatorname{conv}\left(\left\{\alpha a_{0}\right\} \cup A\right)$ with $\left\|z-\alpha a_{0}\right\|>\lambda$, then $\|z\|>\alpha\left\|a_{0}\right\|+\mu$.

Choose $y \in X^{*}$ separating $\left\|a_{0}\right\| U$ from $A$ such that $\left\langle a_{0}, y\right\rangle=\left\|a_{0}\right\|$ and $\|y\|=1\left[24\right.$, p. 76]. Let $z \in \operatorname{conv}\left(\left\{\alpha a_{0}\right\} \cup A\right)$ with $\left\|z-\alpha a_{0}\right\|>\lambda$ be otherwise arbitrary. For some $\beta \in[0,1)$ and $a \in A$ we have $z=\beta\left(\alpha a_{0}\right)+(1-\beta) a$. Notice that $\left\|z-\alpha a_{0}\right\|=(1-\beta)\left\|a-\alpha a_{0}\right\|$, so that $(1-\beta) 2 \rho>\lambda$. Let $a_{1}=$ $\beta a_{0}+(1-\beta) a$. Since $z=a_{1}-\beta(1-\alpha) a_{0}$ and since $a_{1} \in A$, we have

$$
\begin{aligned}
\|z\| & =\|z\| \cdot\|y\| \geq\langle z, y\rangle=\left\langle a_{1}, y\right\rangle-\beta(1-\alpha)\left\langle a_{0}, y\right\rangle \\
& \geq\left\|a_{0}\right\|-\beta(1-\alpha)\left\|a_{0}\right\| \\
& =\alpha\left\|a_{0}\right\|+(1-\beta)(1-\alpha)\left\|a_{0}\right\| \\
& >\alpha\left\|a_{0}\right\|+[\lambda(1-\alpha) / 2 \rho] \cdot\left\|a_{0}\right\| .
\end{aligned}
$$

This shows that $\varphi_{\theta}+I\left(\cdot, \operatorname{conv}\left(\left\{\alpha a_{0}\right\} \cup A\right)\right)$ is well-posed.

It is known that if for each $A \in \mathscr{C}(X)$ the associated metric projection program $\varphi_{\theta}+I(\cdot, A)$ is well-posed, then $X$ is an $E$-space in the sense of Holmes [24, $\S 31]: X$ is reflexive, its unit ball $U$ is rotund, and the norm of $X$ is a Kadec norm. We have generically 
Theorem 5.4. Let $X$ be a reflexive Banach space, with $\mathscr{C}(X)$ equipped with $\tau_{a w}$. Then for each $x \in X,\left\{A \in \mathscr{C}(X): \varphi_{x}+I(\cdot, A)\right.$ is well-posed $\}$ is a dense and $G_{\delta}$ subset of $\mathscr{C}(X)$.

Proof. For each $n \in Z^{+}$, let $\mathscr{F}_{n}=\{A \in \mathscr{C}(X)$ : for each $\alpha>d(x, A)$, we have $\operatorname{diam}\{a \in A:\|x-a\| \leq \alpha\} \geq 1 / n\}$. Notice that $\{a \in A:\|x-a\| \leq \alpha\}$ is nothing but $\operatorname{lev}\left(\varphi_{x}+I(\cdot, A) ; \alpha\right)$. By Lemma 3.2, Theorem 3.6, and Lemma 5.2, each $\mathscr{F}_{n}$ is $\tau_{a w}$-closed in $\mathscr{C}(X)$. The result now follows from Lemma 5.3.

Corollary 5.5. Let $X$ be a separable reflexive Banach space. Then for most $A \in\left\langle\mathscr{C}(X), \tau_{a w}\right\rangle, \varphi_{x}+I(\cdot, A)$ is well-posed for most $x \in X$.

Proof. This follows from Theorem 5.4 and the Kuratowski-Ulam Theorem [27, p. 247], which in this application only requires separability of $X\left(\left\langle\mathscr{C}(X), \tau_{a w}\right\rangle\right.$ is not separable unless $X$ is finite dimensional [3]).

For related genericity results on metric projections, the reader may consult [22].

\section{REFERENCES}

1. E. Asplund and R. T. Rockafellar, Gradients of convex functions, Trans. Amer. Math. Soc. 139 (1969), 443-467.

2. H. Attouch, Variational convergence for functions and operators, Pitman, Boston, Mass., 1984.

3. H. Attouch, R. Lucchetti, and R. Wets, The topology of the $\rho$-Hausdorff distance, Ann. Mat. Pura Appl. (to appear).

4. H. Attouch and R. Wets, Quantitative stability of variational systems: I. The epigraphical distance, Trans. Amer. Math. Soc. (to appear).

5. __ Quantitative stability of variational systems; III. Stability of $\varepsilon$-minimizers, Working paper IIASA, Laxenburg, Austria, 1988.

6. D. Azé and J-P. Penot, Operations on convergent families of sets and functions, Optimization 21 (1990), 521-534.

7. G. Beer, On Mosco convergence of convex sets, Bull. Austral. Math. Soc. 38 (1988), 239-253.

8. On the Young-Fenchel transform for convex functions, Proc. Amer. Math. Soc. 104 (1988), 1115-1123.

9. Convergence of continuous linear functionals and their level sets, Arch. Math. $\mathbf{5 2}$ (1989), 482-491.

10. Conjugate convex functions and the epi-distance topology, Proc. Amer. Math. Soc. 108 (1990), 117-126.

11. __ Metric projections and the Fell topology, Boll. Un. Mat. Ital. (7) 3-B (1989), 925-937.

12. _ Three characterizations of the topology of Mosco convergence for convex functions, Arch. Math. 55 (1990), 285-292.

13. G. Beer and R. Lucchetti, Minima of quasi-convex functions, Optimization 20 (1989), 581596.

14. J. Borwein and S. Fitzpatrick, Mosco convergence and the Kadec property, Proc. Amer. Math. Soc. 106 (1989), 843-850.

15. C. Castaing and M. Valadier, Convex analysis and measurable multifunctions, Lecture Notes in Math., vol. 580, Springer-Verlag, Berlin, 1975. 
16. L. Contesse and J.-P. Penot, Continuity of polarity and conjugacy for the epi-distance topol$o g y$, preprint.

17. F. DeBlasi and J. Myjak, On the minimum distance to a closed convex set in a Banach space, Bull. Acad. Sci. Polon. 29 (1981), 373-376.

18. $\ldots$, Some generic properties in convex and nonconvex optimization theory, Ann. Soc. Math. Polon. 24 (1984), 1-14.

19. N. Efimov and S. Stechkin, Approximative compactness and Chebyshev sets, Soviet Math. Dokl. 2 (1961), 1226-1228.

20. S. Francaviglia, A. Lechicki, and S. Levi, Quasi-uniformization of hyperspaces and convergence of nets of semicontinuous multifunctions, J. Math. Anal. Appl. 112 (1985), 347-370.

21. M. Furi and A. Vignoli, About well-posed optimization problems for functionals in metric spaces, J. Optim. Theory Appl. (1970), 225-229.

22. P. G. Georgiev, The strong Ekeland variational principle, the strong drop theorem, and applications, J. Math. Anal. Appl. 131 (1988), 1-21.

23. J. Giles, Convex analysis with application in differentiation of convex functions, Research Notes in Math., no. 58, Pitman, London, 1982.

24. R. Holmes, $A$ course in optimization and best approximation, Lecture Notes in Math., vol. 257, Springer-Verlag, New York, 1972.

25. J. Joly, Une famille de topologies sur l'ensemble des fonctions convexes pour lesquelles la polarité est bicontinue, J. Math. Pures Appl. 52 (1973), 421-441.

26 . V. Klee, Some new results on smoothness and rotundity in normed linear spaces, Math. Ann. 139 (1959), 51-63.

27. K. Kuratowski, Topology, Vol. 1, Academic Press, New York, 1966.

28. R. Lucchetti, Some aspects of the connections between Hadamard and Tyhonov wellposedness of convex programs, Boll. Un. Mat. Ital. Anal. 1 (1982), 337-344.

29. _ Hadamard and Tyhonov well-posedness in optimal control, Methods Oper. Res. 45 (1983), 113-125.

30. R. Lucchetti and F. Patrone, Sulla densità e genericità di alcuni problemi di minimo ben posti, Boll. Un. Mat. Ital. 15B (1978), 225-240.

31. R. Lucchetti, G. Salinetti, and R. Wets, Uniform convergence of probability measures: topological criteria; preprint.

32. U. Mosco, Convergence of convex sets and of solutions of variational inequalities, Adv. in Math. 3 (1969), 510-585.

33. _ On the continuity of the Young-Fenchel transform, J. Math. Anal. Appl. 35 (1971), 518-535.

34. F. Patrone, Most convex functions are nice, Numer. Funct. Anal. Optim. 9 (1987), 359-369.

35. J. Revalski, Generic well-posedness in some classes of optimization problems, Acta Univ. Carolin. Math. Phys. 28 (1987), 117-125.

36. R. T. Rockafellar, Convex analysis, Princeton Univ. Press, Princeton, N.J., 1970.

37. R. T. Rockafellar and R. Wets, Variational systems, an introduction, Multifunctions and Integrands, G. Salinetti, Ed., Lecture Notes in Math., vol. 1091, Springer-Verlag, Berlin, 1984.

38. G. Salinetti and R. Wets, On the convergence of sequences of convex sets in finite dimensions, SIAM Rev. 21 (1979), 18-33.

39. Y. Sonntag, Convergence au sens de Mosco; théorie et applications à l'approximation des solutions d'inéquations, Thèse d'Etat, Université de Provence, Marseille, 1982. 
40. A. Tyhonov, On the stability of the functional optimization problem, U.S.S.R. Comput. Math. and Math. Phys. 6 (1966), 28-33.

41. R. Wijsman, Convergence of sequences of convex sets, cones, and functions II, Trans. Amer. Math. Soc. 123 (1966), 32-45.

Department of Mathematics, California State University, Los Angeles, California 90032

Department of Mathematics, University of California, Davis, California 95616 